\title{
REMOTE LABORATORY AS CONCEPTUAL MODEL OF BLENDED LEARNING
}

\author{
Antoni Kozic \\ Vilniaus kolegija/University of Applied Sciences, Lithuania \\ Eugenijus Macerauskas \\ Vilniaus kolegija/University of Applied Sciences, \\ Vilnius Gediminas Technical University, Lithuania \\ Paulius Sakalys \\ Vilniaus kolegija/University of Applied Sciences, Lithuania
}

\begin{abstract}
Practice shows that the best built and maintain by the knowledge, is based on and secured personal work experience. Source of expertise can be carried out e-laboratory theoretical and practical experiments. The study key is the development of the system will hamper its inappropriate use. There is a lack of e-tools that enable students to deal with the challenge, problem or phenomenon monitoring experiments $t$. $y$. entering or selecting the initial data. New technology, especially electronic, progress is very important because it allows the development of continuing education, distance education, the democratization of it, adapting to people with different capabilities and needs. Discussed problem is creating a different type of laboratory. The key novelty is that it is not a virtual laboratory based on software tools, but the real remote-controlled electronics lab. Produced simplified conceptual model for remote laboratory experiments and demonstration. The article describes a real remote e-laboratory designed and applied in Vilniaus kolegija/University of Applied Sciences as an experimental practical method for distance learning.
\end{abstract}

Keywords: NI-ELVIS, remote laboratory, blended learning.

\section{Introduction}

To be competitive, strong and outstanding, we need other ways of thinking and training tools that are promising emergence of new technologies and specialties, as well as the basis of new technologies, to convey the classical knowledge (Beghi et al., 2015). In order to promote students' knowledge, the formation of scientific thinking and practical skills - you need to choose the optimal means to achieve that objective. New technologies rapidly and aggressively penetrate not only into working environment, but also to the scientific process. The world around us is changing very fast, cutting-edge technology complexity requires ever more precise instruments. Therefore progression hand in hand with the latest technologies and their deployment makes us viable and unrivalled scientific process and classical laboratories can 
give a new qualitative level working at e-learning ideas. Now it's in the world relevant and widely discussed topic. E-Laboratory conceived as blended learning component - medium where you can learn from remote workstations, self-made laboratory work. You should be able to not only self-made, but also theoretically analyse the results, electronic means of communication to consult with colleagues view previously done similar work to compare results. It was determined that the decreasing number of students - regular training efficiency will decrease. To increase e-learning, remote assessment of the importance of adaptive agent technology influence and blended learning Fig. 1 (Borodin et al., 2009). These guidelines include complete descriptions of the fonts, spacing, and related information for producing your proceedings manuscripts. This template provides authors with most of the formatting specifications needed for preparing electronic versions of their papers. Introduction should include topicality of the subject, the study aims and methods (Barrero et al., 2008; National Instruments, 2005).

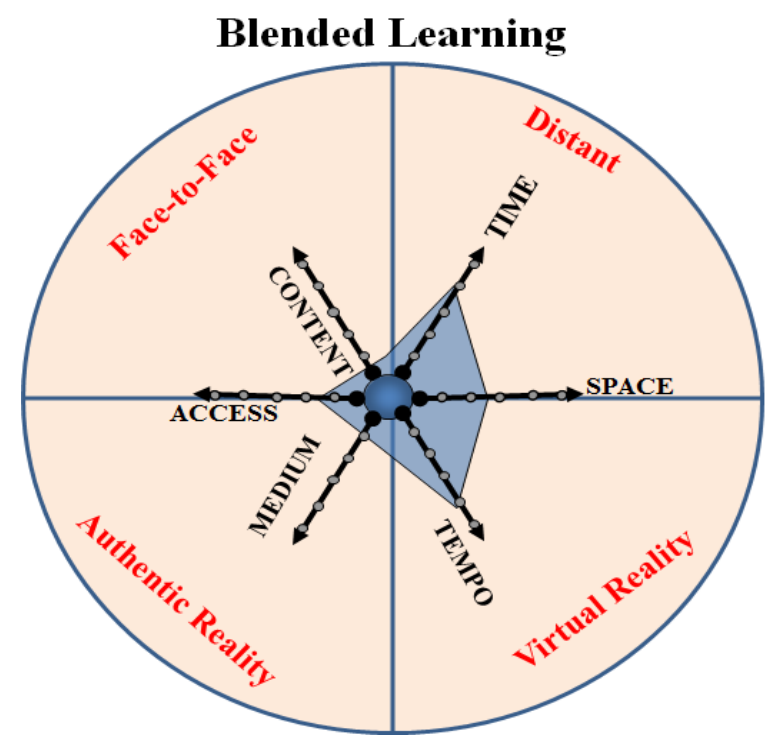

Figure 1 Blended learning scheme

The latest research focuses on sources of information that are now increasingly needed such as study tools that will allow students to construct knowledge through active participation, rather than just information transfer process (IIE Center for Academic Mobility Research, 2014). Therefore it has to operate dynamically changing environment. Most often students apply knowledge to improve understanding of the figures, which is proven to improve visualization. It also created the most complicated structures of the websites that provide interactivity. However, none of these methods does not encourage active student activities that knowledge gained or reinforces skills. The fundamental study development of the system will hamper its use in inadequate and rigid 
thinking, absence of altruism and the pursuit of personal gain. Missing etools that will allow students to solve the problem, the problem or monitor the phenomenon that is experimenting entering or selecting the original data. New technology, particularly electronic, progress has been very important because it allows for the development of continuing education, distance education, democratizing it, adapting to people with different capabilities and needs. Experimental method for the lack of modern distance learning systems - this is just one of the strong arguments in favour of creating remote electronics laboratory to be organized in a practical and laboratory works.

The basic idea is the use of remote control devices and the creation of software packages that may help realize management. Authors decided to use NI ELVIS II (National Instruments Educational Laboratory Virtual Instrumentation Suite), which is used in conjunction with LabVIEW (Laboratory Virtual Instrumentation Engineering Workbench). The purpose is to reveal the educational technology opportunities as possible to classical electronics lab work to provide a qualitative level (Stefanovic et al, 2011).

\section{NI ELVIS educational system}

Remote laboratory is a real remotely controlled laboratory designed to carry out laboratory experiments remotely. The purpose of the laboratory and the relationship with customers (students and teachers) is analogous to the traditional laboratory (Gontean et al., 2009).

Remote operation of the laboratory is based on NI ELVIS II educational devices (Borodin et al., 2009), which together with the LabVIEW environment and specially designed software, works like classical electronics laboratory. The other part is a Java applet acting VNC - TCP/IP protocol (Pengfei \& Luhua, 2009), which is carried out in the laboratory on the server and the user's Internet browser. Moodle learning management system (LMS), which is a virtual educational environment Electronic course is jointly managed by users, connections to laboratories and stores the results of the laboratory experiments (Ursutiu et al., 2004). The client side only a web browser supporting Java applet. No other software tool is needed on the client side, including licenses. Simplified remote laboratory operation in the chart - Fig. 2. Remote laboratory running Client - Java applet - LabVIEW principle. User (student) via a Web browser connects to the LMS (connection with LMS), which is a training course with laboratory experiments tasks. Student select a laboratory experiment and LMS calls the appropriate Java applet (applet call), which is generated dynamically from LabVIEW virtual instrument (VI) laboratory experiment control program. 


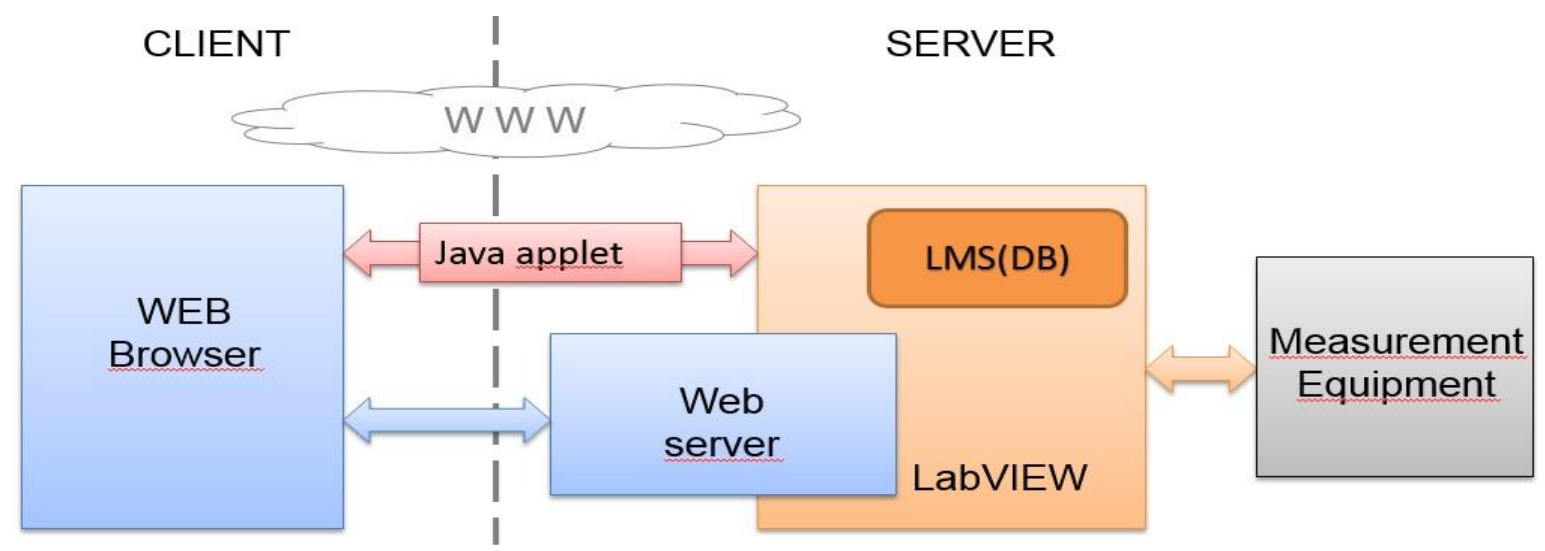

Figure 2 Simplified remote operation of laboratories diagram.

The most Java applet does not perform any action, but only transmits the laboratory experiment image to the web browser, where the user owns a laboratory experiment, as if working directly with VI. All the commands transmitted to VI, and all reactions are shown in the browser. In this way, if applet creates a channel through which passed VI management team. The laboratory experimental data obtained from the LabVIEW VI (experimental management programs) are transmitted to the FMC specially designed storage and database (DB) - the location where the student has carried out together with the identification data. If necessary, the student can obtain a copy of the data to your computer for the further analysis, teachers can access and use them for student assessment.

\section{Laboratory operation}

A detailed remote laboratory operation illustrates the activity diagram in Fig. 3. The diagram shows a laboratory experimental activities process. The diagram to distinguish four operating components that carry out certain actions or laboratory experiment in relation to this:

User - teacher or student joining the lab remotely,

Web server with LMS - this is a web server running LMS,

Lab. server - this lab server running laboratory experiments program designed with LabVIEW environment and experimental equipment owned by the NI ELVIS II,

The experimental device - its LabVIEW controlled test device, which carries out the physical measurements and carry out laboratory experiments on the physical level. 


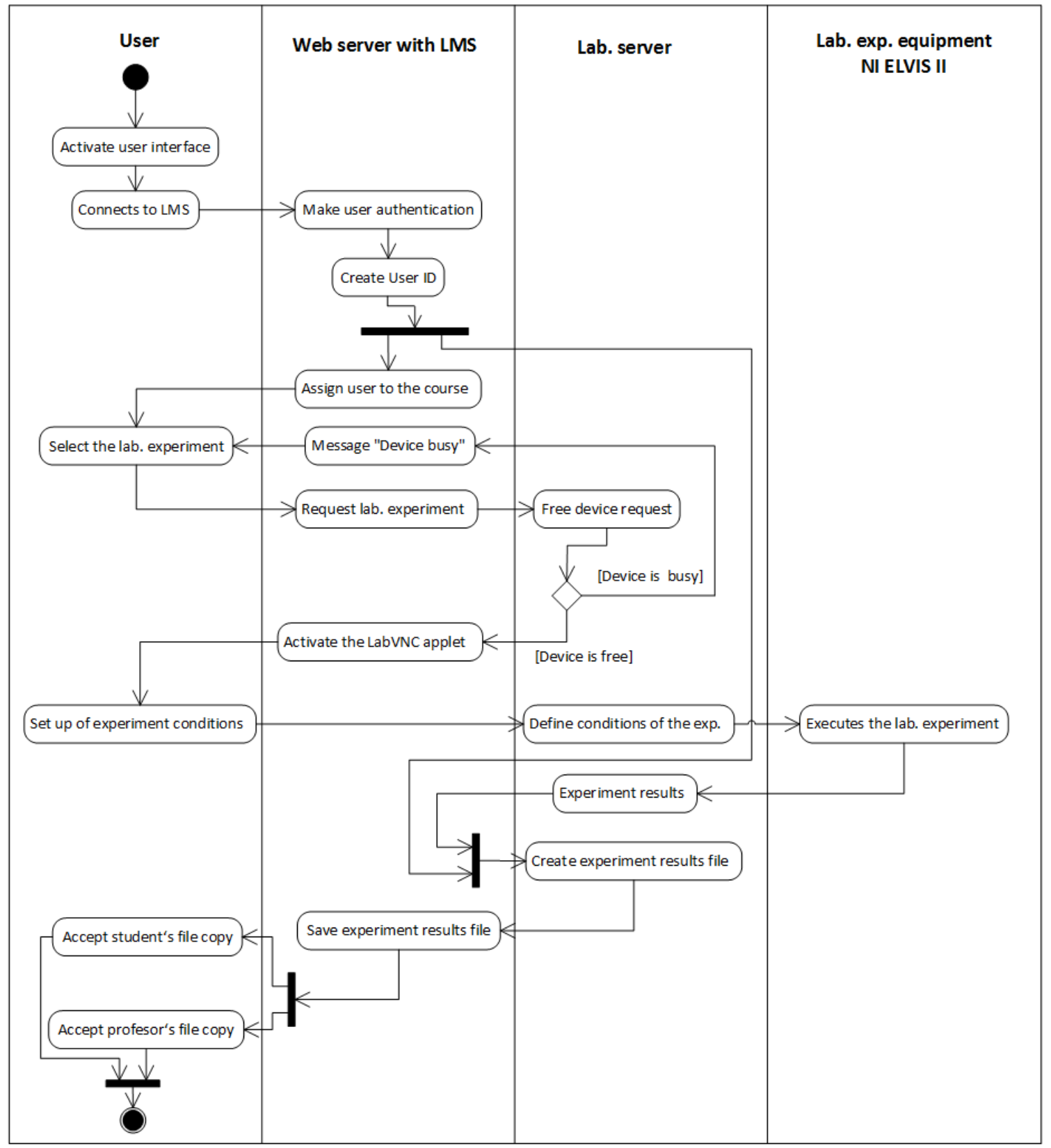

Figure 3 Remote laboratory activity diagram

Remote laboratory is realized in such a principle that student's jobs are independent of the measuring equipment layout. This allows you to make use of computerized measuring instruments that operate managed LabVIEW-based software (Hercog et al., 2007). Such structure is based on laboratory examined the remote NI ELVIS training device management capabilities and practical authors work done (Uran et al., 2007). Laboratory realization is illustrated in Fig. 4. 
Antoni Kozic, Eugenijus Macerauskas, Paulius Sakalys. Remote Laboratory as Conceptual Model of Blended Learning

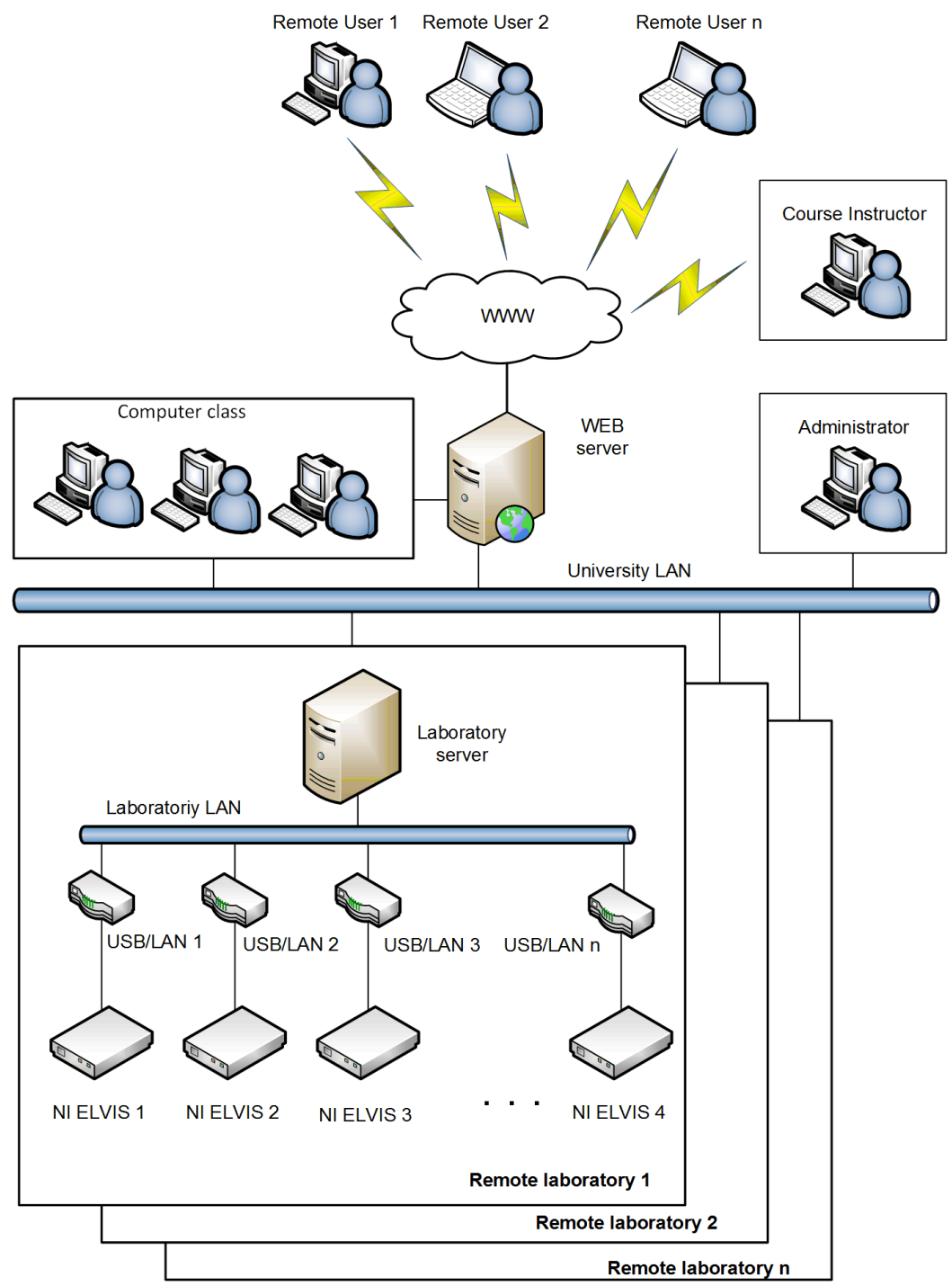

Figure 4 Hardware remote laboratory system realization scheme

Measuring laboratories may be several types, which are divided according to the equipment used, rather than the nature of the experiments carried out as a classic case of laboratories. Each laboratory measurement consists of measuring devices ELVIS_1, ELVIS_2, ELVIS_3 ... ELVIS_n, which replaces physical jobs. All these devices are specialized and intended specifically for teaching computer-laboratory experiments (National Instruments, 2010). Measuring devices are controlled from a common measurement server and connect to the server via a USB-LAN converter which converts the USB interface data transfer protocol in the TCP / IP protocol without any changes in software (Travis, 2009). In this way, each test device has a separate IP address that the election of the server communicates with the device. USB-LAN converter operates Anywhere USB and the principle of bi-directional signal transmits only the required device, no matter what the data is (Digi Inc., 2016). 


\section{Experimental results}

One of the usual laboratory performed laboratory experiment variation Diodes comparison study of the properties "is presented as an illustration previously described remote operation of the laboratory. During the Moodle LMS accessible laboratory experiment work window, which is also a laboratory experiment tasks and report - Fig 5. The experiment was prepared by a similar method to the classical laboratory (Fabregas et al., 2011).

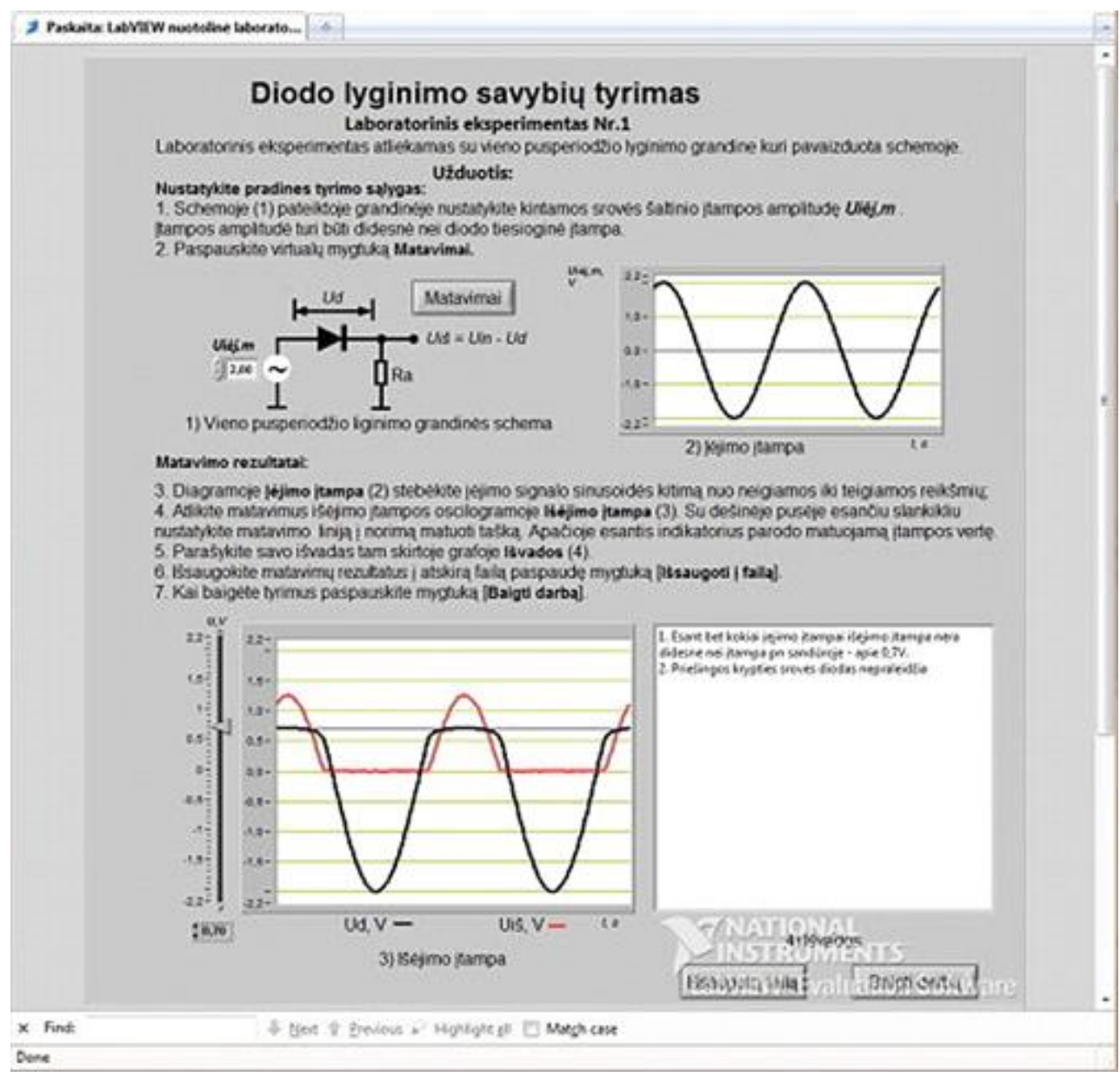

Figure 5 Through Moodle accessible "Investigation of rectifying diode"

Experimental short window is a task that contains text form and labeled Item 1 - 7, specifically what the student has to be carried out (1). Initial experimental conditions are determined by the selection of the input voltage value simply power the circuit diagram (2). Input circuit initial conditions - the input signal is visible oscillogram (6). After selecting the voltage measurements carried out by pressing a virtual button. Continue to monitor the results of the measurements the oscillogram (4) in which a student can measure the voltage 
level using a virtual meter (3). After performing the experiment, student signature of its findings directly to the laboratory experiment work window, the purpose-built field (6) where the data is stored as text information. After performing the experiment, student clicks the button to save the file and all measurements along with a student identification data, the experiment timing mark and the student's conclusions text saved in a text file.

\section{Conclusions}

Summing up the above-described remote laboratory, which reflects the practical Moodle, LabVIEW and NI ELVIS II, LabVNC aspects of the application and a Java applet, which is realized through a remote control experiment, it is possible to realize a real remote-controlled laboratory. The essential feature of such a laboratory in improving the quality of education is that the student laboratory can achieve anytime, anywhere, and thus becomes a sort of laboratory Toolbox tool where knowledge acquired through experimentation.

In the event of direct competition between universities in the market will remain those who are able to provide students with state of the art, easy to understand and useful not only theoretical but also practical knowledge. The essence of the system is that NI technology makes it easy to create any measurement devices and complexes, adapting them to the curriculum, rather than by contraries. The system allows you to give up not only many measuring devices, but also the laboratory working paper version, because all the tasks performed on the computer screen. Universal National Instruments' educational experiment stands NI ELVIS II., who has shown excellent results in the electronics lab, remote electronics lab concept that is successfully achieved both from a local college network as well as online. The implementation of this project would be fully equipped electronics laboratory experiments, remote system, allowing for both a variety of realistic simulation experiments and tests of physical objects in a network environment. Summing up the remote electronics lab as blended learning is concept model that reflects the practical LabVIEW and NI ELVIS II aspects of the application that can realize the remote electronics lab. The biggest advantage of such type of laboratory in improving the quality of education is that the student in the lab can reach at any time and from anywhere.

The laboratory becomes the Toolbox tool where knowledge acquired through experimentation. Distance learning with real e-laboratory enables almost unlimited expand access to higher education for students, ensuring virtually unlimited dissemination of scientific information are needed to accelerate the search for information and saves time. The wise use of them 
provides opportunities for radical studies and research processes, renewal and innovation, and process management using a blended learning concept.

\section{References}

Barrero, F., Toral, S. \& Gallardo, S. (2008). eDSPLab: Remote Laboratory for Experiments on DSP Applications. Internet Research, 18(1), 79 - 92.

Beghi, A., Cervato, A. \& Rampazzo, M. (2015). A Remote Refrigeration Laboratory for Control Engineering Education, IFAC-PapersOnUne, 48(29), 25-30.

Borodin, S., Batovrin, V. \& Romanov, A. (2009). LabVIEW Elektronikos praktiniai darbai (pp.20-25). Learning materials: VPU Press

Digi Inc. (2016). USB and Serial Connectivity solutions AnywhereUSB: a global survey Downloaded from http://www.digi.com/pdf/prd_usb_anywhereusb.pdf

Fabregas, E., Farias, G., Dormido-Canto, S., Dormidoa, S. \& Esquembre F. (2011). Developing a Remote Laboratory for Engineering Education. Computers \& Education, 57, 1686-1697.

Gontean, A., Szab, R. \& Lie, I. (2009). LabVIEW Powered Remote Lab, Design and Technology of Electronics Packages. SIITME 2009 15th International Symposium for, p.17-20, Sept. 2009.

Hercog, D., Gergic, B., Uran, S. \& Jezernik, K. (2007). A DSP-Based Remote Control Laboratory. IEEE Transactions on Industrial Electronics, 54(6), 3057 - 3068.

Nantional Instruments (2005). NI Educational Laboratory Virtual Instrumentation Suite II Series (NI ELVIS II Series) User Manual: a global survey. Downloaded from http://www.ni.com/pdf/manuals/374629c.pdf

National Instruments. 2010). LabVIEW Web UI Builder Help 2010. Available from http://zone.ni.com/reference/en-XX/help/373286A-01/

Pengfei, L. \& Luhua, N. (2009). Remote Control Laboratory Based On LabVIEW. IEEE 2 nd International Conference on Intelligent Computation Technology and Automation, v.4, p.84-87, oct.2009. ISBN 978-0-7695-3804-4.

Stefanovic, M., Cvijetkovic, Matijevic, M. \& Simic, V. (2011). A LabVIEW-based Remote Laboratory Experiments for Control Engineering Education. Computer Applications in Engineering Education. 19(3), 538-549.

The IIE Center for Academic Mobility Research and Impact Institute of International Education (2014). Fall 2014 Snapshot survey of international student enrolment: a global survey. Downloaded from http://www.iie.org/ /media/Files/Corporate/OpenDoors/Special-Reports/Fall-2014-International-Student-Enrollment-Survey.pdf?la=en

Travis, J. (2010). Internet Applications in LabVIEW. USA: Hall PTR.

Uran, S., Hercog, D., Jezernik, K. (2007). Remote Control Laboratory with Moodle Booking System, Industrial Electronics. IEEE International Symposium, p.2978-2983, june 2007. ISBN: 978-1-4244-0754-5.

Ursutiu, D., Cotfas, P., Samoila, C., Zamfira, S. \& Auer M. (2004). NI-ELVIS in Remote Electronic Laboratory REL. Proceedings of the $1^{\text {st }}$ International Symposium on Remote Engineering and Virtual Instrumentation, Villach, Austria, 28./29. september 2004. ISBN 3-89958-090-7. 